\title{
The Evolution of Copper in the Globular Cluster $\omega$ Centauri
}

\author{
Katia Cunha \\ Observatório Nacional, Rua General José Cristino 77, 20921-400, São Critóvão, RJ, Brazil; \\ katia@on.br \\ Verne V. Smith \\ Department of Physics, University of Texas El Paso, El Paso, TX 79968 USA; \\ verne@barium.physics.utep.edu \\ Nicholas B. Suntzeff \\ Cerro Tololo Interamerican Observatory, Casilla 603, La Serena, Chile; nsuntzeff@noao.edu \\ John E. Norris \\ Research School of Astronomy \& Astrophysics, The Australian National University, Mt. Stromlo \\ Observatory, Cotter Road, Weston, ACT 2611, Australia; jen@mso.anu.edu.au \\ Gary S. Da Costa \\ Research School of Astronomy \& Astrophysics, The Australian National University, Mt. Stromlo \\ Observatory, Cotter Road, Weston, ACT 2611, Australia; gdc@mso.anu.edu.au \\ Bertrand Plez \\ GRAAL, Universite Montpellier II, cc072, 34095 Montpellier cedex 05, France; \\ plez@graal.univ-montp2.fr
}

\begin{abstract}
Copper abundances are presented for 40 red-giant members of the massive Galactic globular cluster $\omega$ Centauri, as well as 15 red-giant members of the globular clusters NGC288, NGC362, NGC3201, NGC6752, and M4 (NGC6121). The spectra are of relatively high spectral-resolution and signal-to-noise. Using these abundances, plus published literature values for field stars, the abundance trends of $[\mathrm{Cu} / \mathrm{Fe}]$ are defined as a function of $[\mathrm{Fe} / \mathrm{H}]$. The lowest metallicity stars in $\omega$ Cen have $[\mathrm{Fe} / \mathrm{H}] \sim-2.0$, with the stars in this sample spanning a range from $[\mathrm{Fe} / \mathrm{H}] \sim-2.0$ to -0.8 . In the field star sample, $[\mathrm{Cu} / \mathrm{Fe}]$ rises from about -0.8 , at $[\mathrm{Fe} / \mathrm{H}]=-2.5$, to about -0.4 at $[\mathrm{Fe} / \mathrm{H}] \sim-1.4$, and then rises rapidly to $[\mathrm{Cu} / \mathrm{Fe}] \sim 0.0$ at $[\mathrm{Fe} / \mathrm{H}]=-1.1$. The globular clusters (other than $\omega$ Cen) tend to also follow the trend as displayed by the field stars. Unlike the field stars, however, $\omega$ Cen displays a constant ratio of $[\mathrm{Cu} / \mathrm{Fe}] \sim-0.5$ all the way to $[\mathrm{Fe} / \mathrm{H}]=-0.8$. At the metallicity of $[\mathrm{Fe} / \mathrm{H}]=-0.8$, the values of $[\mathrm{Cu} / \mathrm{Fe}]$ in $\omega$ Cen fall below the corresponding mean ratio in the field stars by roughly $0.5 \mathrm{dex}$. If copper is produced primarily in type Ia supernovae, as suggested in the literature, the lack of an increase in $[\mathrm{Cu} / \mathrm{Fe}]$ in $\omega$ Cen would suggest very little contribution from SN Ia to its chemical evolution within the metallicity range from $[\mathrm{Fe} / \mathrm{H}]$ of -2.0 up to -0.8 .
\end{abstract}


Subject headings: globular clusters: individual ( $\omega$ Centauri; NGC288; NGC362; NGC3201; NGC6752; M4) 


\section{Introduction}

Omega Centauri is the most massive globular cluster known in the Milky Way. This cluster is also the only one known whose stars display a large range of abundances in all elements, such as $\mathrm{Fe}, \mathrm{Ca}, \mathrm{Ti}, \mathrm{Ni}$ or $\mathrm{O}$. The abundance of a standard metallicity indicator, such as $\mathrm{Fe}$, spans a range from $[\mathrm{Fe} / \mathrm{H}] \sim-2.0$ up to -0.4 (Pancino et al. 2000). The abundance variations arise from chemical self-enrichment and evolution in $\omega$ Cen, with many of these elements produced in supernovae of type II (SN II), e.g. as discussed by Brown et al. (1991) or Brown \& Wallerstein (1993). In addition to self-enrichment in elements produced in SN II, $\omega$ Cen displays an even larger abundance range in the neutron-capture s-process elements, such as Y, Zr, Ba, or La. Ratios, such as $[\mathrm{Ba} / \mathrm{Fe}]$, increase tremendously as $[\mathrm{Fe} / \mathrm{H}]$ increases in $\omega$ Cen: this cluster underwent an enormous increase in s-process abundances during its chemical evolution, with the s-process elements being produced in asymptotic giant branch (AGB) stars (Lloyd Evans 1983).

Other elements, produced in sites other than SN II or AGB stars, can provide additional insights into the chemical evolution and possible progenitor identity of this relatively small stellar system. In this study, we report on copper abundances in 40 red giant stars in $\omega$ Cen, (10 of which have already been studied in Smith et al. 2000), as well as in small samples of red giants in the other globular clusters NGC288, NGC362, NGC3201, NGC6752, and M4 (NGC6121). Copper abundances in these various globular cluster giants are then compared to samples of field stars from the literature, in order to explore the behavior of $\mathrm{Cu}$ with metallicity in the Galaxy. The $[\mathrm{Cu} / \mathrm{Fe}]$ ratio is not constant in halo stars, as shown by Sneden \& Crocker (1988) and Sneden et al. (1991), where $[\mathrm{Cu} / \mathrm{Fe}]$ increases with increasing metallicity as $[\mathrm{Cu} / \mathrm{Fe}] \alpha 0.4[\mathrm{Fe} / \mathrm{H}]$ up to $[\mathrm{Fe} / \mathrm{H}]=-1$, where it then reaches the solar ratio. For higher metallicities, the $[\mathrm{Cu} / \mathrm{Fe}]$ is solar. These trends were seen both in field and globular cluster stars.

Given the somewhat complicated chemical history of copper, both Sneden et al. (1991) and Matteucci et al. (1993) reviewed the nucleosynthetic sites of copper (as well as zinc). Sneden et al. suggested that much of the copper in metal-poor stars was formed in the weak component of the s-process, whereby a neutron capture on Fe-peak elements is produced during the late stages of core He-burning (Couch et al. 1974, Raiteri, et al. 1991) in massive stars. Some copper was also expected to be formed in explosive nucleosynthesis from SN II. At higher metallicities, they expect that most of the copper was formed in Type Ia supernovae (SN Ia). Only a small fraction ( $<30 \%$ ) of the copper abundance in the sun can come from s-process without gross inconsistencies with other elemental and isotopic abundances (Raiteri et al. 1992). Because the weak component of the s-process is a secondary mechanism for nucleosynthesis, this site for the formation of copper agrees qualitatively with a relationship of increasing $[\mathrm{Cu} / \mathrm{Fe}]$ as a function of $[\mathrm{Fe} / \mathrm{H}]$ in metal-poor stars.

Matteucci et al. (1993) revised the Sneden et al. (1991) model to force a quantitative fit to the data. As they note, any model is hampered by the poorly understood nucleosynthetic yields of $\mathrm{Cu}$ in SN II and SN Ia. The conclusion of their paper is that adequate fits to these elements 
in metal-poor stars could be made only if there was significant nucleosynthesis from SN Ia in the metallicity range $-2<[\mathrm{Fe} / \mathrm{H}]<-1$. One theoretical prediction that indirectly pertains to the production of copper via SN Ia is the study by Kobayashi et al. (1998) who have revisited the models for the formation of Type Ia supernovae. In the models of single degenerate progenitors, the wind from the accreting white dwarf plays an important role in regulating the slow increase in mass of the white dwarf. The existence of this wind allows a much larger parameter space of single degenerate progenitors to produce Type Ia supernova explosions. Their prediction is that SN Ia will not form for $[\mathrm{Fe} / \mathrm{H}]<-1$, apparently contradicting the models of Matteucci et al. (1993).

The origins of the metallicity variations and the details of chemical evolution in $\omega$ Cen are not understood. However, it clearly has a different chemical evolution history from the Galactic halo and disk. Moreover, little observational work on copper has been published since Sneden et al. (1991). With this in mind, we set out to explore the copper abundances in $\omega$ Cen with its different chemical history to gain insight into the sites of copper nucleosynthesis.

\section{Observations}

The echelle spectra assembled and analyzed in this paper were obtained at two different telescopes: the Blanco $4 \mathrm{~m}$ at CTIO and the 3.9m Anglo-Australian Telescope (AAT). The CTIO spectra were obtained using the cassegrain echelle spectrograph and the Red Long camera (590 $\mathrm{mm}$ focal length). Typical resolutions were 35,000 with wavelength coverage from about 5235 $8518 \AA$. In this project, 10 red-giant members of $\omega$ Cen were observed, along with 4 members each in NGC288 and M4, 3 members in NGC362, and 2 members each in NGC3201 and NGC6752. The $\omega$ Cen giant spectra were analyzed in Smith et al. (2000), while the other cluster members are analyzed for the first time in this paper. All red giants observed have effective temperatures in the range of roughly $3800-4500 \mathrm{~K}$. The CTIO data were reduced to wavelength-calibrated spectra with the IRAF programs. More details in the data reduction procedures are described in Smith et al. (2000). Final signal-to-noise ratios in these spectra were typically 100 to 250 .

In addition to the CTIO spectra, high-resolution $(\mathrm{R} \sim 38,000)$ spectra were analyzed for $\mathrm{Cu}$ I from the spectra previously studied by Norris \& Da Costa (1995) and Norris, Da Costa, \& Tingay (1995). These two papers provided equivalent widths and an abundance analysis of several elements for a sample of $40 \omega$ Cen giants, but the authors did not determine $\mathrm{Cu}$ abundances. These spectra were obtained with the University College London Echelle Spectrograph (UCLES), with a CCD detector at the coude focus of the $3.9 \mathrm{~m}$ Anglo Australian Telescope (AAT). The data were reduced to 1-d spectra using both IRAF and FIGARO routines: more detailed discussion of the data reduction of the UCLES spectra can be found in Norris et al. (1995). Typical signal-to-noise ratios of these final, reduced spectra are $\mathrm{S} / \mathrm{N} \sim 50$.

Examples of spectra showing the $\mathrm{Cu}$ I line, as well as their synthetic fits, are discussed and shown in Section 3 (Analysis). Table 1 is a summary of the observing log for the spectra analyzed 
in this study.

\section{Analysis}

The techniques for model atmosphere abundance analyses of red giants spanning the effective temperature range sampled in this study are well established and more detailed discussions of the techniques adopted here can be found in earlier papers: e.g., Norris \& Da Costa (1995), Smith et al. (1995), or Smith et al. (2000). In short, published model atmospheres, based upon the Bell et al. (1976: here BEGN) grid for red giants, are used with basic stellar parameters $\left(T_{\text {eff }}, \log g\right.$, and metallicity) derived from a combination of photometry and spectroscopic analyses.

The abundance analysis was carried out for the program stars under the assumption of LTE, using the most recent version of the spectral analysis code MOOG (Sneden 1973). Model atmospheres were interpolated within the standard grid of BEGN models produced by Gustafsson et al. (1975). For red giants spanning the range of temperatures, gravities, and metallicities that represent the program stars, use of these models, with appropriately selected samples of spectral lines, can produce abundance results that are accurate (on an absolute scale) to within typically $0.1-0.2$ dex, depending upon the spectral lines and species in question.

The copper abundances derived in this study are based on the single $\mathrm{Cu}$ I line at $5782.127 \AA$, and adopting the gf-value used by Sneden \& Crocker (1988) (same as in Sneden, Gratton, \& Crocker 1991), which itself is from the laboratory determination by Hannaford \& Lowe (1983). In addition, hyperfine (hfs) and isotopic splitting is significant for this line and these components were included in the analysis, with the hfs and isotopic data taken from Biehl (1976). The various hyperfine and isotopic components of the $5782 \AA$ line, along with their gf-values, are listed in Table 2: the gf-values are weighted by their respective solar system isotopic fractions of 0.69 for ${ }^{63} \mathrm{Cu}$ and 0.31 for ${ }^{65} \mathrm{Cu}$.

A synthetic fit of the $\mathrm{Cu}$ I line in the Solar Flux Atlas (Kurucz et al. 1984) is shown in Figure 1. The overall fit of the synthetic hfs profile with the observed solar line-profile is quite good, although there are some small mismatches near the line center (these will not affect measurably the derived stellar $\mathrm{Cu}$ abundances). The resulting solar $\mathrm{Cu}$ abundance is $\log \epsilon=4.06$, compared to the accepted photospheric abundance of $4.21 \pm 0.04$ (Grevesse \& Sauval 1998). It should be noted that the solar $\mathrm{Cu}$ abundance quoted by Grevesse \& Sauval was derived using the gf-value from Koch \& Richter (1968), with log $(\mathrm{gf})_{5782}=-1.78$ compared to -1.72 from Hannaford \& Lowe (1983). This suggests that a comparison of the Grevesse $\mathrm{Cu}$ photospheric abundance to the one derived here should include a -0.06 dex offset from the differing gf-values. Also, Grevesse quoted a result based upon the empirical Holweger-Müller model (Holweger \& Müller 1974), while we used a theoretical MARCS model: the small remaining difference of 0.09 dex is, thus, entirely reasonable. Of importance for later discussion comparing results derived here with the field-star abundances from Sneden \& Crocker (1988) and Sneden et al. (1991), is that these studies and our 
study used the same gf-value for the $5782 \AA \mathrm{Cu}$ I line. In their analysis, Sneden \& Crocker (1988) derived a solar photospheric copper abundance using the same gf-value as adopted here, and found $\log \epsilon=4.12$. They used the Holweger-Müller solar model atmosphere and the small difference of 0.06 dex between these respective studies can be attributed to different solar models and slightly different microturbulent velocities $\left(0.8 \mathrm{~km} \mathrm{~s}^{-1}\right.$ in Sneden \& Crocker and $1.0 \mathrm{~km} \mathrm{~s}^{-1}$ here).

Derived abundances depend upon the adopted values of effective temperature $\left(T_{\text {eff }}\right)$, surface gravity (parameterized by $\log g$ ), overall stellar metallicity (typified by $[\mathrm{Fe} / \mathrm{H}]$ ), and the microturbulent velocity $(\xi)$. In the Smith et al. (2000) analysis, the effective temperatures and surface gravities were derived from spectroscopy: the spectroscopic analysis used the numerous Fe I and Fe II lines (with well-determined laboratory $g f$-values) measurable in high-resolution spectra. Demanding the simultaneous conditions of zero slope in plots of Fe abundance (from Fe I) versus both excitation potential and equivalent width yield the effective temperature and microturbulent velocity. Enforcing ionization equilibrium, such that both Fe I and Fe II yield the same abundance, provides the model surface gravity. Norris \& da Costa (1995) used a different approach to derive stellar parameters, with the effective temperatures determined from the infrared photometry of Persson et al. (1980) and Paltoglou \& Norris (1989), using the T eff $^{-I R}$ color scales from Cohen, Frogel, \& Persson (1978).

A comparison of the derived stellar parameters in the two independent studies (Norris \& Da Costa 1995 and Smith et al. 2000) is useful and is possible for 4 stars in common to both samples: ROA's 102, 213, 253, and 219. Mean differences and standard deviations of the basic stellar parameters, in the sense of 'Smith et al. minus Norris \& Da Costa', were computed and these are found to be: $\Delta \mathrm{T}_{\text {eff }}=+25 \pm 125 \mathrm{~K}, \Delta(\log \mathrm{g})=-0.02 \pm 0.25 \mathrm{dex}, \Delta \xi=+0.10 \pm 0.27 \mathrm{~km} \mathrm{~s}^{-1}$, and $\Delta[\mathrm{Fe} / \mathrm{H}]=-0.05 \pm 0.06$ dex. Therefore, there are no significant offsets between the stellar parameters derived by Norris \& Da Costa (1995) and those from Smith et al. (2000), with the scatter, as measured by the standard deviations, being very close to the estimated uncertainties as discussed in both papers.

An additional check of the Smith et al. (2000) Fe I- $\mathrm{T}_{\text {eff }}$ scale against IR color scales is possible using data from the Two Micron All Sky Survey (2MASS: http://www.ipac.caltech.edu/2mass/). Infrared $\mathrm{J}, \mathrm{H}$, and $\mathrm{K}$ magnitudes can be found for 8 of the Smith et al. stars and effective temperatures were computed for these giants using four different $(\mathrm{V}-\mathrm{K})-\mathrm{T}_{\text {eff }}$ calibrations, from Cohen et al. (1978), McWilliam (1990), van Belle et al. (1999), and Alonso, Arribas, \& Martinez-Roger (1999). Transformations of the 2MASS colors, to other photometric systems, can be found in Carpenter (2001) and, for the $\omega$ Cen stars, are quite small. The four different $(\mathrm{V}-\mathrm{K})$ calibrations yield effective temperatures with scatters of about $\pm 60 \mathrm{~K}$ for an inidividual $\omega$ Cen giant, and the comparison of the mean difference and standard deviation of $\mathrm{T}_{\text {eff }}(\mathrm{Fe} \mathrm{I})-\mathrm{T}_{\text {eff }}(\mathrm{V}-\mathrm{K})$ is $+38 \pm 138 \mathrm{~K}$ : this is very similar to the comparison to the Norris \& Da Costa (1995) T Teff's. Due to the lack of any significant offsets, we expect no systematic differences as the previously derived stellar parameters from Norris \& Da Costa and Smith et al. are on a consistent scale within the errors. Therefore, the adopted parameters and Fe abundances for the $\omega$ Cen sample stars remain 
the same from the previous studies and these are assembled in Table 3 together with the derived copper abundances for the sample stars studied in this cluster.

In addition, the other sample stars from the globular clusters NGC's 288, 362, 3201, 6752, and M4 are analyzed such that their stellar parameters are derived in the same way as by Smith et al. (2000) and therefore rely on the spectral lines of Fe I and Fe II present in their spectra. Tables 4 and 5 list the Fe I and Fe II measured equivalent widths for these 15 giants (as they have not been published previously). Their corresponding stellar parameters and resulting $\mathrm{Fe}$ and $\mathrm{Cu}$ abundances are presented in Table 6.

We note that some of the stars from our globular clusters sample have been examined previously (using the same spectra) by Ivans et al. (1999), for M4, and Gonzalez \& Wallerstein (1998), for NGC3201 (with neither study including copper in their analyses). These authors used either a different technique (Ivans et al. used line-depth ratios), or a somewhat different set of Fe I and Fe II lines (Gonzalez \& Wallerstein 1998) to derive parameters. A comparison of their adopted parameters is useful as an indication of the magnitude of the uncertainties involved in different spectroscopic determinations. For the four M4 giants, differences of (This Study - Ivans et al.) results in $\Delta \mathrm{T}_{\text {eff }}=+105 \pm 43 \mathrm{~K}, \Delta(\log \mathrm{g})=-0.06 \pm 0.16 \mathrm{dex}, \Delta \xi=+0.02 \pm 0.10 \mathrm{~km} \mathrm{~s}^{-1}$, and $\Delta([\mathrm{Fe} / \mathrm{H}])=+0.04 \pm 0.07$. The comparison between (This Study - Gonzalez \& Wallerstein) finds (with only average differences listed, as there are only 2 stars in the comparison) $\Delta \mathrm{T}_{\text {eff }}=+125 \mathrm{~K}$, $\Delta(\log \mathrm{g})=+0.25 \mathrm{dex}, \Delta \xi=+0.10 \mathrm{~km} \mathrm{~s}^{-1}$, and $\Delta([\mathrm{Fe} / \mathrm{H}])=-0.08$ dex.

In general terms, these various comparisons between independent studies indicate that, for these red giants, effective temperatures can be defined to within $\sim 100 \mathrm{~K}$, surface gravities to about 0.1-0.2 dex, microturbulent velocities within $0.1-0.2 \mathrm{~km} \mathrm{~s}^{-1}$, and metallicities to within about 0.05 dex. In terms of copper abundances, typical errors of $100 \mathrm{~K}$ in $\mathrm{T}_{\text {eff }}, 0.2 \mathrm{dex}$ in $\log \mathrm{g}$, and $0.2 \mathrm{~km}$ $\mathrm{s}^{-1}$ in $\xi$ would lead to expected uncertainties of 0.18 dex in derived $\mathrm{Cu}$ abundances.

Sample spectral fits of the $\mathrm{Cu}$ I line regions are shown in Figures 2, 3, and 4. The star pe75 from NGC362 is shown in Figure 2, illustrating one of the globular cluster giants (other than $\omega$ $\mathrm{Cen}$ ) observed from CTIO. Note that the $\mathrm{Cu} \mathrm{I}$ line is blended with a $\mathrm{Cr}$ I line, although the $\mathrm{Cu}$ I line itself is well-defined. Synthetic spectra are plotted for three different $\mathrm{Cu}$ abundances: the best-fit value, plus-or-minus 0.1 dex values. Figure 3 also shows a CTIO spectrum, but for the $\omega$ Cen star ROA 324 (a star with a rather low $[\mathrm{Cu} / \mathrm{Fe}]$ ratio). Finally, Figure 4 shows an AAT spectrum and resulting synthetic fits for the $\omega$ Cen giant ROA 252 (with $[\mathrm{Fe} / \mathrm{H}]=-1.74$ ).

Because some of the $\omega$ Cen giants are fairly cool ( $\mathrm{T}_{\text {eff }}$ as low as $\left.3750 \mathrm{~K}\right)$, a check on the model atmospheres was performed using the latest version of the MARCS code, OSMARCS (Plez, Brett, \& Nordlund 1992; Edvardsson et al. 1993; Asplund et al. 1997), that contains more extensive and up-to-date molecular opacities. An OSMARCS model was computed for the coolest giant, ROA201, and the $\mathrm{Cu}$ I region was synthesized and analyzed with the newer model as a comparison to the older MARCS model. Virtually no differences were found in the respective synthetic spectra and the same copper abundance was found from both models. This exercise demonstrates that 
over the temperature range of giants studied here the $\mathrm{Cu}$ abundance scale is insensitive to the older MARCS versus newer OSMARCS atmospheres.

As a final check on the copper and iron abundance presented here, possible systematic effects, especially with temperature, were investigated. In low-mass red-giant branches (RGB's), the position of the giant branch in a $T_{\text {eff }}$-luminosity plane is a function of metallicity, with the more metal-rich red giants being cooler. Since both the Norris \& Da Costa (1995) and Smith et al. (2000) samples were observed, essentially, at near-constant V-magnitudes while crossing the RGB in color, the more metal-rich stars tend to be cooler in both samples. Such a correlation could lead to systematic effects in $[\mathrm{Cu} / \mathrm{Fe}]$ versus $[\mathrm{Fe} / \mathrm{H}]$. Possible systematic trends correlated with stellar parameters are investigated via Figure 5. In the top panel is a spectroscopically derived HR-Diagram, with $\log g$ versus $\mathrm{T}_{\text {eff }}$ : it is clear that the lower surface gravity stars tend to be cooler, as would be expected for low-mass stars ascending the RGB. Superimposed on the observationally derived points are two sample isochrones from Bergbusch \& Vandenberg (1992) for 12 Gyr populations with $[\mathrm{Fe} / \mathrm{H}]=-2.0$ and -1.0 , respectively. These iron abundances should nearly bracket the $\omega$ Cen stars and, indeed, this is the case. The close match between the derived stellar parameters and those from stellar models suggests that there are not large systematic offsets in the temperatures and gravities used to derive the abundances.

The middle panel of Figure 5 shows $[\mathrm{Fe} / \mathrm{H}]$ versus $\mathrm{T}_{\text {eff }}$ and demonstrates that the coolest red giants in both samples tend to be found among the most metal-rich stars (with these $[\mathrm{Fe} / \mathrm{H}]$ values being those derived spectroscopically): this is the expected result. Finally, the bottom panel shows $[\mathrm{Cu} / \mathrm{Fe}]$ versus effective temperature and no significant trend exists. The straight line is a least-squares fit to the points and has but an insignificant non-zero slope; there is no evidence that there are measurable systematic abundance trends created by the derivation of stellar parameters or the abundance analysis. It should be noted that if $\omega$ Cen stars followed the behavior of the halo one would expect a trend, i.e. the more metal rich (and cooler) giants would have larger values of $[\mathrm{Cu} / \mathrm{Fe}]$. It would be unlikely that a systematic temperature error would just conspire to provide the observed zero slope of $[\mathrm{Cu} / \mathrm{Fe}]$ with effective tempetarure. It is more likely that the stellar parameters are not affected by significant systematic errors and that the $[\mathrm{Cu} / \mathrm{Fe}]$ ratio in $\omega$ Cen is quite constant from $[\mathrm{Fe} / \mathrm{H}]=-2.0$ to -0.8 . A combination of all of the $[\mathrm{Cu} / \mathrm{Fe}]$ values for the $\omega$ Cen stars results in a mean and standard deviation of $-0.50 \pm 0.11$ : this indicates no evolution in the copper to iron yields over much of the chemical evolution within $\omega$ Cen.

\section{Discussion}

The abundances of copper discussed in this paper are assembled in the different panels of Figure 6 as $[\mathrm{Cu} / \mathrm{Fe}]$ versus $[\mathrm{Fe} / \mathrm{H}]$. As a reference point, we adopt $\log \epsilon(\mathrm{Fe})=7.50$ and $\log \epsilon(\mathrm{Cu})=$ 4.06 as the corresponding solar abundances. All three samples of stars are shown in Figure 6: the field star results, the "mono-metallicity" globular clusters (NGC's 288, 362, 3201, 6752, and M4), and $\omega$ Cen. 
In the top panel of Figure 6 are shown the results from Sneden \& Crocker (1988) and Sneden et al. (1991) for the field stars, with their corresponding error estimates in $[\mathrm{Cu} / \mathrm{Fe}]$ and $[\mathrm{Fe} / \mathrm{H}]$ plotted. The straight lines are least-squares fits to two metallicity regimes $([\mathrm{Fe} / \mathrm{H}] \leq-1$ and $[\mathrm{Fe} / \mathrm{H}] \geq-1$ ) and are meant only to serve as guides to the eye in order to more easily identify the behavior of copper with metallicity $([\mathrm{Fe} / \mathrm{H}])$. It is found that $[\mathrm{Cu} / \mathrm{Fe}]$ is effectively constant in the high-metallicity regime while clearly decreasing towards lower metallicities in those stars having $[\mathrm{Fe} / \mathrm{H}] \leq-1.4$ (as discussed in Sneden et al. 1991), with an approximate slope of 0.4 in $[\mathrm{Cu} / \mathrm{Fe}]$ versus $[\mathrm{Fe} / \mathrm{H}]$.

The middle panel of Figure 6 again shows the field-star results (with error bars suppressed for clarity), upon which are plotted our abundances for the globular clusters M4, NGC's 288, 362,3201 , and 6752 . Note that the individual scatter, in both $[\mathrm{Fe} / \mathrm{H}]$ and $[\mathrm{Cu} / \mathrm{Fe}]$, within each globular cluster is quite small; thus, for example, the small differences in $[\mathrm{Cu} / \mathrm{Fe}]$ between NGC362 and the clusters M4 and NGC288, at similar values of $[\mathrm{Fe} / \mathrm{H}]$, are probably real, with the three studied stars in NGC 362 falling slightly below the straight line that represents the field behavior. (Although there is a gap in the sample field population at roughly this metallicity). Except for NGC362, that may have a somewhat low $[\mathrm{Cu} / \mathrm{Fe}]$ ratio for its value of $[\mathrm{Fe} / \mathrm{H}]$, the other 4 globular clusters fall quite close to the mean trend of the field stars.

The general trend for the $\omega$ Cen stars is shown in the bottom panel of Figure 6, again with the field-star results overplotted for comparison. Unlike the field, the $\omega$ Cen members show a constant $[\mathrm{Cu} / \mathrm{Fe}]$ ratio of about -0.5 in the interval from $[\mathrm{Fe} / \mathrm{H}]$ of $\sim-2.0$ up to -0.8 : there may be a hint that the scatter in $[\mathrm{Cu} / \mathrm{Fe}]$ increases towards the highest metallicities. The values of $[\mathrm{Cu} / \mathrm{Fe}]$ in the field halo stars and $\omega$ Cen are in agreement at $[\mathrm{Fe} / \mathrm{H}] \sim-2$, where the two samples overlap, but are clearly different at $[\mathrm{Fe} / \mathrm{H}] \sim-1$, with the $\omega$ Cen stars falling below the field in $[\mathrm{Cu} / \mathrm{Fe}]$. In particular, over the upper range of metallicities of the $\omega$ Cen sample studied here (with $-1.2 \leq[\mathrm{Fe} / \mathrm{H}] \leq-0.8$ ) the mean value of $[\mathrm{Cu} / \mathrm{Fe}]$ and standard deviation is $-0.52 \pm 0.17$ (for 9 stars). For the same $[\mathrm{Fe} / \mathrm{H}]$ range in the field-star sample, $[\mathrm{Cu} / \mathrm{Fe}]=-0.05 \pm 0.18$ (for 8 stars). The nearly horizontal line in this panel is a least-squares fit to the $\omega$ Cen points that has a near-zero slope. The 8-pointed stars are the results for $\omega$ Cen from Pancino et al. (2002), and include two members that are significantly more metal-rich than those in our sample. For the stars with $[\mathrm{Fe} / \mathrm{H}] \leq-0.8$, the Pancino et al. (2002) results overlap those found in this study; however, one of the metal-rich Pancino et al. (2002) stars (with $[\mathrm{Fe} / \mathrm{H}] \sim-0.5$ ) has a $[\mathrm{Cu} / \mathrm{Fe}]$ value that is nearly equal to those found in the field stars at this metallicity. A more rigorous comparison between $\omega$ Cen and field behavior will be aided by an increased sample of galactic field stars allowing for a complete elucidation of the behavior of copper in the metallicity range between $[\mathrm{Fe} / \mathrm{H}]-2.0$ and -0.5 .

From the point of view of observations, the behavior of $[\mathrm{Cu} / \mathrm{Fe}]$ is different between the field halo stars and the stars in $\omega$ Cen over the studied metallicity range. Can this different behavior in the two samples be understood within some simple context? It is safe to assume that the production site(s) for $\mathrm{Cu}$ involve either massive stars and Type II supernovae, or Type 
Ia supernovae (which result from binary-star evolution), and do not involve nucleosynthesis in low-mass AGB stars. Within this context, $\omega$ Cen shows clear evidence of enormous elemental contributions from low-mass AGB stars via s-process nucleosynthesis (e.g. Norris \& Da Costa 1995), thus, the observed constant values of $[\mathrm{Cu} / \mathrm{Fe}]$ found here do not fit a picture in which $\mathrm{Cu}$ synthesis arises in AGB stars. Addressing the point of whether $\mathrm{Cu}$ production is associated with either SN II or SN Ia, there are conflicting ideas. As mentioned in the Introduction, based on their observed trend of $[\mathrm{Cu} / \mathrm{Fe}] \alpha 0.4[\mathrm{Fe} / \mathrm{H}]$, Sneden et al. (1991) favor a production model for $\mathrm{Cu}$ in metal-poor stars $([\mathrm{Fe} / \mathrm{H}] \leq-1.0)$ which has two sources, both occurring in massive stars: the weak s-process, operating during core He burning, plus the e-process of Si burning during Type II supernovae. In the more metal-rich disk stars, an additional source of $\mathrm{Cu}$ appears from Type Ia supernovae. On the other hand, Matteucci et al. (1993) use the $\mathrm{Cu}-$ Fe relation from Sneden et al. (1991) to reach somewhat different conclusions about the origin of $\mathrm{Cu}$. Their chemical models are best fit with $\mathrm{Cu}$ being produced predominantly in Type Ia supernovae, even down to metallicities well below -1.0 in $[\mathrm{Fe} / \mathrm{H}]$. One of the main points of their study is to assume that there is already a SN Ia contribution between metallicities $[\mathrm{Fe} / \mathrm{H}]-2.0$ and -1.0 .

A number of previous studies of $\omega$ Cen (e.g. Brown \& Wallerstein 1993; Norris \& Da Costa 1995; Smith et al. 1995, 2000) find that elements such as Si, Ca, or Ti exhibit the same behavior as observed in the halo field, i.e. that their abundances are enhanced relative to Fe. This is interpreted to result from chemical enrichment from Type II supernovae. Since the halo field and $\omega$ Cen are identical in this respect, there is evidence that $\omega$ Cen was enriched by SN II ejecta. Thus, the fact that $[\mathrm{Cu} / \mathrm{Fe}]$ exhibits different behavior between the field-halo stars and $\omega$ Cen could suggest that the main source of $\mathrm{Cu}$ within this metallicity range is not massive stars. Of course, one could speculate that, for example, the weak s-process operates over some restricted mass range and that the mass function of $\omega$ Cen was deficient in stars over this range. This speculation, however, introduces additional parameters and will not be explored further here.

If the $[\mathrm{Cu} / \mathrm{Fe}]$ ratios in $\omega$ Cen do indeed eliminate massive stars as the dominant $\mathrm{Cu}$ producers, this leaves Type Ia supernovae, as suggested by Matteucci et al. (1993). This winnowing of Cu sources still leaves the problem of why the field halo and $\omega$ Cen exhibit different behaviors of $[\mathrm{Cu} / \mathrm{Fe}]$ versus $[\mathrm{Fe} / \mathrm{H}]$, but there are a number of possible explanations: 1) The timescale for SN Ia progenitor evolution exceeds the timescale of chemical evolution within $\omega$ Cen, 2) SN Ia systems do not form in metal-poor environments, as suggested by Kobayahsi et al. (1998), 3) SN Ia progenitor systems (presumed to be binaries) are sufficiently rare, that none would be expected to form in a small system, such as $\omega$ Cen, 4) SN Ia binary progenitor systems form, but are subsequently disrupted in the dense environment of a globular cluster, or, 5) the negligible contribution from SN Ia's over much of $\omega$ Cen's chemical evolution may result from the selective retention of stellar ejecta in low-mass systems (where AGB winds are more efficiently retained than SN II ejecta, and, in the case here, SN Ia ejecta almost not retained at all).

Concerning option (1), photometric studies by Hilker \& Richtler (2000) and Hughes \& Wallerstein (2000), as well as the s-process spectroscopic analyses by Smith et al. (2000), indicate 
that it took $\sim 1-3$ Gyr for $\omega$ Cen to evolve from $[\mathrm{Fe} / \mathrm{H}] \sim-2.0$ to -1.0 . The nucleosynthesis from Type II supernovae and from the weak component of the s-process will happen on time scales of $10^{7}$ years. The timescale for the formation of Type Ia supernovae is not clear mostly due to the lack of a definite progenitor model. The discussion by Kobayashi et al. (1998) gives typical time scales for the double degenerate model as 0.3 Gyr and for single degenerate models of 0.6 Gyrs. A discussion of a larger set of possible Type Ia models gives rough timescales from 0.1 to 4 Gyrs (Branch et al. 1995). It has been long established that the rates of Type Ia supernovae are higher in late-type galaxies (Oemler \& Tinsley 1979) than in early types, which would support a shorter time scale for formation of Type Ia events. It therefore seems safe to assume that the age spread measured in the stellar population $\omega$ Cen is significantly longer than the time needed to form the typical Type Ia supernovae.

Concerning option (2), Kobayashi et al. (1998) develop models for binary SN Ia progenitors in which the wind from the white dwarf plays a key role in determining whether a Chandrasekhar mass limit is reached. The strength of the wind is a function of the metallicity, and Kobayashi et al. (1998) conclude that Type Ia supernovae are quenched below metallicities of -1.0. In this type of picture, it might be expected that SN Ia's would be inhibited in all low metallicity environments and thus the field halo and $\omega$ Cen would exhibit the same type of behavior. However, this is not what is observed. But of course, this contention resides on the assumption that Ia's are the main producers of $\mathrm{Cu}$.

Concerning possibility (3) from above, Pagel (1997) estimates that the SN Ia rate in the Milky Way is 0.33 per century. Taking a Galactic stellar mass of $\sim 10^{11} \mathrm{M}_{\odot}$, this translates to a "mass specific rate" of $3 \times 10^{-14}$ SN Ia events per year per solar mass of stars. Given an $\omega$ Cen protomass of $\sim 10^{7} \mathrm{M}_{\odot}$, it can be estimated that the SN Ia rate in the cluster would be $10^{-7}$ events per year once the time has passed for SN Ia events to turn on. Over some $10^{9}$ years of chemical evolution, $\omega$ Cen would have $\sim 100$ SN Ia's occur. As each SN Ia can eject up to $\sim 0.6 \mathrm{M}_{\odot}$ of Fe (Pagel 1997), these events would have a measurable impact on the abundances, if such ejecta were retained. Of course, this simple argument assumes that the rate of binary formation in a globular cluster is the same as in the Galaxy as a whole. There is no observational evidence concerning the initial fraction of binary systems formed in globular clusters; however, there is evidence that binary sytems can be disrupted in the environment of a globular cluster. In fact, Côté et al. (1996) find that $\omega$ Cen has a smaller fraction of binary systems than other globular clusters studied by them (e.g. M71, M4 and NGC3201 among others). This could be a possible explanation for the lack of SN Ia enrichment over much of the $[\mathrm{Fe} / \mathrm{H}]$ evolution in $\omega$ Cen, as suggested by possibility (4) from above. In this context, perhaps the incidence of Type Ia supernovae is simply lower in $\omega$ Cen compared to the field due to some destruction of SN Ia progenitor systems. This might explain the delayed, but eventual appearence of SN Ia enrichment in the most metal-rich stars in $\omega$ Cen $([\mathrm{Fe} / \mathrm{H}] \sim-0.5)$ as derived by Pancino et al. (2002).

Another possibility (number 5 from above) in understanding these peculiar chemical traits in $\omega$ Cen comes from Smith et al. (2000). The observation that $\omega$ Cen contains such a large s-process 
component led to the speculation that in a relatively low-mass stellar system AGB ejecta, because of their low velocity winds, might be more efficiently retained relative to the much faster moving Type II supernova ejecta. The AGB ejecta would then be mixed with a relatively small amount of SN II ejecta that was retained, as well as with the residual gas of initial composition. The same sort of speculative explanation could hold for explosions of Type Ia supernovae. Differing retention efficiencies between SN II and SN Ia ejecta might be caused by the differing interstellar environments where the explosions occur. For example, Recchi, Matteucci \& D'Ercole (2001) model chemical and dynamical evolution in a low-mass, starburst dwarf galaxy and find that a metal-enriched galactic wind is driven out of the galaxy by SN II and SN Ia explosions. They also find that the SN Ia ejecta are preferentially ejected relative to SN II ejecta. Gnedin et al. (2002) have pointed out, however, that the current escape velocity from $\omega$ Cen is not that different from the other globular clusters. The hypothesis of selective mass loss or retention, on the other hand, applies to the progenitor object (proto $\omega$ Cen) before it was captured into its current retrograde orbit around the Milky Way; the nature of that progenitor object remains unknown. Distinguishing whether possibility (4) or (5) best pertains to understanding the chemical evolution within $\omega$ Cen can probably be constrained by determining abundance ratios in a wide variety of stellar environments, such as other types of small galaxies within the Local Group, as well as in a larger sample of $\omega$ Cen members from the most metal-rich population identified by Pancino et al. (2000).

Our abundance results lead us to the conclusion that the copper to iron ratio in $\omega$ Cen is nearly constant over about a factor of 10-15 range in iron abundance $(-2.0 \leq[\mathrm{Fe} / \mathrm{H}] \leq-0.8)$. Over this same range of $[\mathrm{Fe} / \mathrm{H}]$ in the field stars, $[\mathrm{Cu} / \mathrm{Fe}]$ increases by about 0.5 dex. This demonstrates yet another peculiar trait of the chemical evolution found within $\omega$ Cen when compared to the Galactic halo field stars at comparable metallicities (the other peculiar chemical trait being the large s-process abundance component in $\omega$ Cen).

\section{Conclusions}

In this work we discuss $\mathrm{Cu}$ abundances in 40 red-giant members of $\omega \mathrm{Cen}$, as well as 15 red-giant members of 5 other globular clusters that overlap the range of $[\mathrm{Fe} / \mathrm{H}]$ found in $\omega$ Cen. Abundances from field stars already published in the literature by Sneden \& Crocker (1988) and Sneden et al. (1991) are included in the discussion. It is found that both the field star sample and 4 of the 5 "mono-metallicity" Galactic globular clusters display similar values of $[\mathrm{Cu} / \mathrm{Fe}]$ at a given $[\mathrm{Fe} / \mathrm{H}]$. The $\omega$ Cen members, however, tend to have smaller and nearly-constant values of $[\mathrm{Cu} / \mathrm{Fe}]$ ratios, when compared to the other globulars and the field, as $[\mathrm{Fe} / \mathrm{H}]$ increases. If copper is produced preferentially in SN Ia, relative to SN II, this indicates that $\omega$ Cen stars show little, if any, enrichment from SN Ia's up to a cluster metallicity of $[\mathrm{Fe} / \mathrm{H}] \sim-0.8$. A lag in SN Ia chemical enrichment in $\omega$ Cen could result either from the disruption of binary SN Ia progenitor systems in $\omega$ Cen, or from a higher fraction of SN Ia ejecta being lost from $\omega$ Cen. 
This work is supported in part by the National Science Foundation through AST99-87374 (VVS). 


\section{REFERENCES}

Alonso, A., Arribas, S., \& Martinez-Roger, C. 1999, A\&AS, 139, 335

Asplund, M., Gustafsson, B., Kiselman, D., \& Eriksson, K. 1997 A\&A 318, 521

Bard, A., Kock, A., \& Kock, M. 1991, A\&A, 248, 315

Bell, R.A., Eriksson, K., Gustafsson, B.E., \& Nordlund, A 1976, A\&AS, 23, 37

Bergbusch, P.A., \& Vandenberg, D.A. 1992, ApJS, 81, 163

Biehl, D. 1976, Ph.D. Thesis, Kiel

Branch, D., Livio, M., Yungelson, L.R., Boffi, F.R., \& Baron, E. 1995, PASP, 107, 1019

Brown, J.A., Wallerstein, G., Cunha, K., \& Smith, V.V. 1991, A\&A, 249, L13

Brown, J.A., \& Wallerstein, G. 1993, AJ, 106, 133

Carpenter, J.M. 2001, AJ, 121, 2851

Cohen, J.G., Persson, S.E., \& Frogel, J.A. 1978, ApJ, 222, 165

Couch, R.G, Schmiderkamp, A.B, and Arnett W.D 1974, ApJ, 190, 95

Côté, P., Pryor, C., McClure, R.D., Fletcher, J.M., \& Hesser, J.E. 1996, AJ, 112, 574

Edvardsson, B., Andersen, J., Gustafsson, B., Lambert, D. L., Nissen, P. E., \& Tomkin, J. 1993 A\&A 275, 101

Gnedin, O.Y., HongSheng, Z., Pringle, J.E., Fall, S.M., Livio, M., Meylan, G. 2002, ApJ, in press (20 March issue)

Gonzalez, G., \& Wallerstein, G. 1998, AJ, 116, 765

Grevesse, N., \& Sauval, A.J. 1998, Sp. Sci. Rev. 85, 161

Gustafsson, B.E., Bell, R.A., Eriksson, K., \& Nordlund, A. 1975, A\&A, 42, 407

Hannaford, P., \& Lowe, R.M. 1983, Opt. Engineering, 22, 532

Hilker, M., \& Richtler, T. 2000, A\&A, 362, 895

Holweger, H., \& Müller, E.A. 1974, Solar Physics, 39, 19

Holweger, H., Bard, A., Kock, A., \& Kock, M. 1991, A\&A, 249, 545

Hughes, J., \& Wallerstein, G. 2000, AJ, 119, 1225 
Ivans, I.I., Sneden, C., Kraft, R.P., Suntzeff, N.B., Smith, V.V., Langer, G.E., \& Fulbright, J.P. 1999, AJ, 118, 1273

Kobayashi, C., Tsujimoto, T., Nomoto, K., Hachisu, I., \& Kato, M. 1998,ApJ, 503, L155

Koch, M., \& Richter,J. 1968, Zs. Ap., 69, 180

Kurucz, R.L., Furenlid, I., Brault, J., \& Testerman, L. 1984, Solar Flux Atlas from 296 tp 1300 nm, National Solar Obs., Atlas No. 1 (Harvard University Press, Cambridge)

Lloyd Evans, T. 1983, MNRAS, 204, 975

Matteucci, F., Raiteri, C.M., Busso, M., Gallino, R., \& Gratton, R.G. 1993, A\&A, 272, 421

McWilliam, A. 1990, ApJS, 74, 1075

McWilliam, A. 1997, ARAA, 35, 503

Norris, J.E., \& Da Costa, G.S. 1995, ApJ, 447, 680

Norris, J.E., Da Costa, G.S., \& Tingay, S.J. 1995, ApJS, 99, 637

Oemler, A., \& Tinsley, B.M. 1979, AJ, 84, 985

Paltoglu, G., \& Norris, J.E. 1989, ApJ, 336, 185

Pagel, B.E.J. 1997, Nucleosynthesis and Chemical Evolution of Galaxies (Cambridge University Press: Cambridge).

Pancino, E., Ferraro, F.R., Bellazzini, M., Piotto, G., \& Zoccali, M. 2000, ApJ, 534, L83

Pancino, E., Pasquini, L., Hill, V., Ferraro, F.R., \& Bellazzini, M. 2002, ApJ, in press

Plez, B., Brett, J. M., \& Nordlund, A. 1992, A\&A, 256, 551

Persson, S.E., Cohen, J.G., Matthews, K., Frogel, J.A., \& Aaronson, M. 1980, ApJ, 235, 452

Raiteri, C.M., Busso, M., Picchio, G., Gallino, R., \& Pulone, L. 1991, ApJ, 367, 228

Raiteri, C.M., Gallino, R., Busso, M., Neuberger, D., \& Kaeppeler, F. 1993, ApJ 419, 207

Recchi, S., Matteucci, F., \& D'Ercole, A. 2001, MNRAS, 322, 800

Smith, V.V., Cunha, K., \& Lambert, D.L. 1995, AJ, 110, 2827

Smith, V.V., Suntzeff, N.B., Cunha, K., Gallino, R., Busso, M., Lambert, D.L., \& Straniero, O. 2000, AJ, 119, 1239

Sneden, C. 1973, Ph.D. Thesis, The University of Texas at Austin 
Sneden, C., \& Crocker, D.A. 1988, ApJ, 335, 406

Sneden, C., Gratton, R.G., \& Crocker, D.A. 1991, A\&A, 246, 354

van Belle, G.T., Lane, B.F., Thompson, R.R., Boden, A.F., Colavita, M.M., Dumont, P.J., Mobley, D.W., Palmer, D., Shao, M., Vasisht, G.X., Wallace, J.K., Creech-Eakman, M.J., Koresko, C.D., Kulkarni, S.R., Pan, X.P., Gubler, J. 1999, AJ, 117, 521 
Fig. 1.- Spectral syntheses of the observed Cu I line in the solar flux atlas (Kurucz et al. 1984) for different copper abundances. The synthetic $\mathrm{Cu}$ I line-profile produced from the hyperfine and isotopic components in Table 2 produces a good fit to the observed solar profile for $\log \epsilon(\mathrm{Cu})=4.06$. The synthetic profiles are convolved with broadening from the instrumental profile (as discussed by Kurucz et al. 1984), solar rotation, and required additional broadening, assumed to consist of gaussian distributed macroturbulence $(\Gamma)$.

Fig. 2.- The Cu I spectral region for a red giant in the globular cluster NGC 362. This illustrates one of the CTIO spectra and shows three different copper abundances (each differing by $0.10 \mathrm{dex}$ ) in the synthetic spectra.

Fig. 3.- The $\mathrm{Cu}$ I line in an $\omega$ Cen red giant: this target is one of the most metal-rich stars in this sample $([\mathrm{Fe} / \mathrm{H}]=-0.95)$ and is one of the CTIO specta. Three different copper abundances are shown in the synthetic spectra, illustrating the sensitivity of the $\mathrm{Cu}$ I line-strength to the copper abundance.

Fig. 4.- An example of an AAT spectrum and the synthetic fits. This is a fairly metal-poor red giant in $\omega$ Cen, with $[\mathrm{Fe} / \mathrm{H}]=-1.74$. The typical AAT spectra have signal-to-noise ratios that are somewhat less than in the CTIO spectra (as can be seen by comparing this spectrum to those in Figures 2 and 3), but more stars are contained in the AAT dataset.

Fig. 5.- A search for possible trends in combinations of the derived stellar parameters and abundances. The top panel is a version of an HR-diagram in which surface gravity is plotted versus effective temperature; as red giants of nearly the same mass ascend the giant branch towards lower values of $\mathrm{T}_{\text {eff }}$, their surface gravities should decrease (as observed). The solid curves are sample isochrones (from Bergbusch \& Vandenberg 1992) for 12 Gyr populations having two different metallicities. The middle panel shows derived Fe abundances versus $\mathrm{T}_{\text {eff }}$ : note the trend of lower effective temperatures as $[\mathrm{Fe} / \mathrm{H}]$, which is expected for low-mass red giants (the red-giant branch shifts towards cooler temperatures as metallicity increases). The bottom panel plots $[\mathrm{Cu} / \mathrm{Fe}]$ against $\mathrm{T}_{\text {eff. }}$ This distribution is essentially flat, as illustrated by the fitted least-squares line which has a near-zero slope (there is slight, non-significant slope). There are no trends in the derived copper iron ratios with temperature.

Fig. 6. - The ratios of $[\mathrm{Cu} / \mathrm{Fe}]$ versus $[\mathrm{Fe} / \mathrm{H}]$ for three samples of stars: the field stars from Sneden \& Crocker (1988) and Sneden et al. (1991) are shown as open circles in all three panels, while members of the mono-metallicity globular clusters NGC's 288, 362, 3201, and 6752, and M4 are plotted in the middle panel and the $\omega$ Cen giants are shown in the bottom panel. The straight lines through the field stars are linear least-squares fits to two separate regimes $([\mathrm{Fe} / \mathrm{H}] \leq-1.1$ and $\geq-1.1$. The field-star results show no significant evolution in $[\mathrm{Cu} / \mathrm{Fe}]$ versus $[\mathrm{Fe} / \mathrm{H}]$ in the high-metallicity sample, but show decreasing $[\mathrm{Cu} / \mathrm{Fe}]$ with decreasing $[\mathrm{Fe} / \mathrm{H}]$ in the low-metallicity sample (with a slope in this type of plot of 0.4). The mono-metallicity globular cluster stars shown in the middle panel generally fall well within the scatter in $[\mathrm{Cu} / \mathrm{Fe}]$ exhibited by the field stars. 
The cluster NGC 362 may be slightly different, falling slightly below the field-star distribution: this cluster deserves future attention. The copper to iron ratios for $\omega$ Cen are shown in the bottom panel, and exhibit a very flat distribution of $[\mathrm{Cu} / \mathrm{Fe}]$ over the metallicity range covered by the sample of stars analyzed here (with $[\mathrm{Fe} / \mathrm{H}]=-2.0$ up to -0.8). This behavior is different from the field stars over this metallicity range, which show increasing $[\mathrm{Cu} / \mathrm{Fe}]$ over this same range in $[\mathrm{Fe} / \mathrm{H}]$. The more metal-rich giants in $\omega$ Cen, analyzed recently by Pancino et al. (2002), also tend to follow this flat distribution of $[\mathrm{Cu} / \mathrm{Fe}]$, except for the two most metal-rich members, which show increasing copper to iron ratios. 


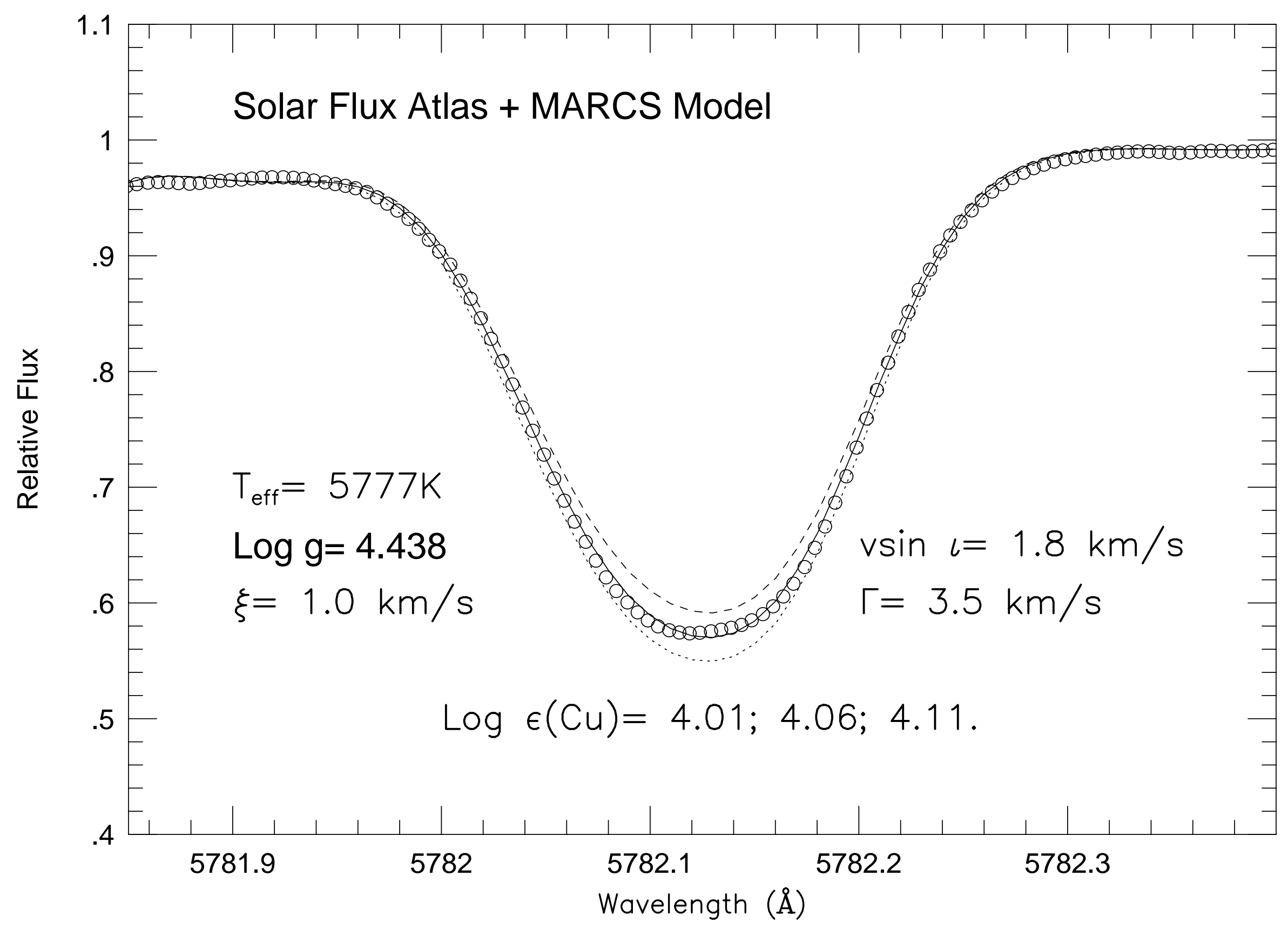


TABLE 1

DATA LOG

\begin{tabular}{cccc}
\hline \hline Cluster & Number of Stars & Dates & Observatory \\
\hline$\omega$ Cen & 10 & $1994 \& 1997$ & CTIO \\
& 30 & $1992 \& 1993$ & AAT \\
M4 & 4 & 1991 & CTIO \\
NGC288 & 4 & 1995 & CTIO \\
NGC362 & 3 & 1995 & CTIO \\
NGC3201 & 2 & 1991 & CTIO \\
NGC6752 & 2 & 1991 & CTIO \\
\hline
\end{tabular}




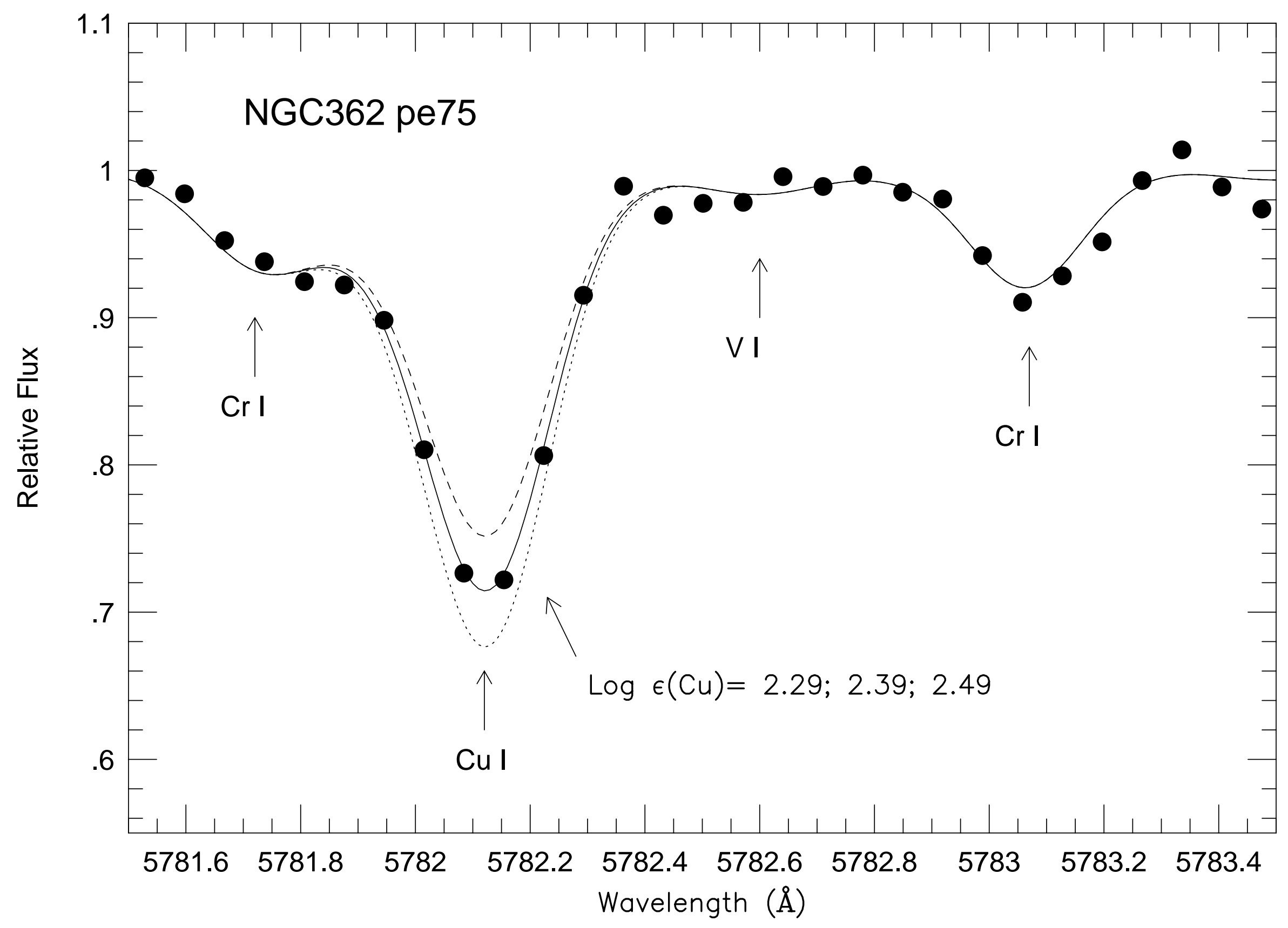


TABLE 2

Copper Spectral-Line Component Structure

\begin{tabular}{ccc}
\hline \hline Wavelength $(\AA)$ & Isotope & gf-Value \\
\hline 5782.032 & ${ }^{65} \mathrm{Cu}$ & $3.698 \mathrm{e}-04$ \\
5782.042 & ${ }^{65} \mathrm{Cu}$ & $1.841 \mathrm{e}-04$ \\
5782.054 & ${ }^{65} \mathrm{Cu}$ & $9.247 \mathrm{e}-04$ \\
5782.064 & ${ }^{63} \mathrm{Cu}$ & $8.222 \mathrm{e}-04$ \\
5782.073 & ${ }^{63} \mathrm{Cu}$ & $4.083 \mathrm{e}-04$ \\
5782.084 & ${ }^{63} \mathrm{Cu}$ & $2.051 \mathrm{e}-03$ \\
5782.086 & ${ }^{65} \mathrm{Cu}$ & $9.247 \mathrm{e}-04$ \\
5782.098 & ${ }^{65} \mathrm{Cu}$ & $9.247 \mathrm{e}-04$ \\
5782.113 & ${ }^{63} \mathrm{Cu}$ & $2.051 \mathrm{e}-03$ \\
5782.124 & ${ }^{63} \mathrm{Cu}$ & $2.051 \mathrm{e}-03$ \\
5782.153 & ${ }^{65} \mathrm{Cu}$ & $2.588 \mathrm{e}-03$ \\
5782.173 & ${ }^{63} \mathrm{Cu}$ & $5.741 \mathrm{e}-03$ \\
& & \\
\hline
\end{tabular}

Note.- Total value is $\mathrm{gf}=1.995 \mathrm{e}-02$. Isotopic composition is assumed to be that of the solar system: ${ }^{63} \mathrm{Cu}=69 \%,{ }^{65} \mathrm{Cu}=31 \%$. The lower excitation energy of this level is $\chi=1.642$ $\mathrm{eV}$. 


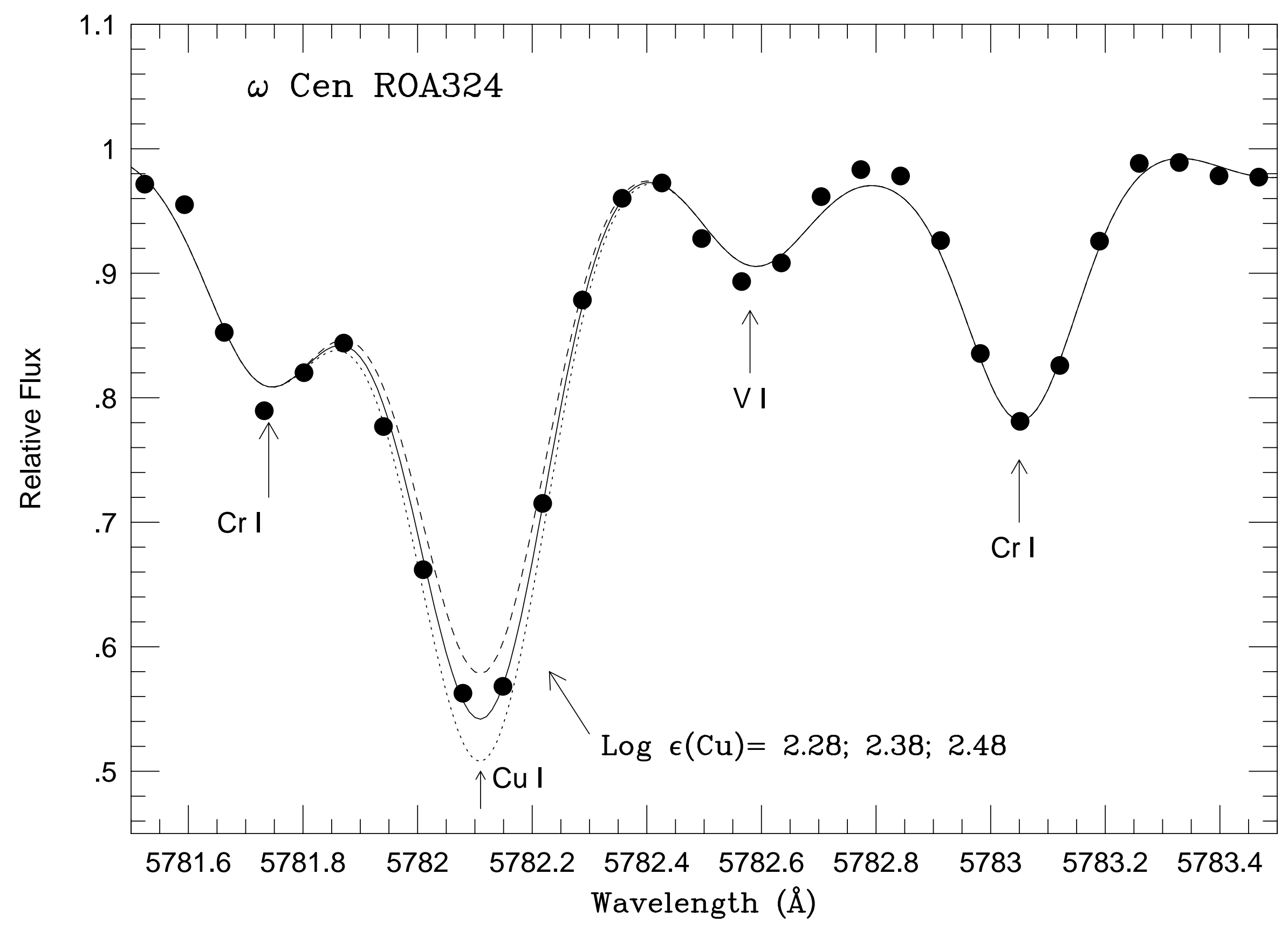


TABLE 3

$\omega$ Cen Stars: Adopted Parameters \& Abundances.

\begin{tabular}{|c|c|c|c|c|c|c|}
\hline Star & Obs. & $\mathrm{T}_{\mathrm{eff}}(\mathrm{K})$ & $\log g\left(\mathrm{~cm} \mathrm{~s}^{-2}\right)$ & $\xi\left(\mathrm{km} \mathrm{s}^{-1}\right)$ & $\mathrm{A}(\mathrm{Fe})$ & $\mathrm{A}(\mathrm{Cu})$ \\
\hline ROA40 & AAT & 4200 & 0.5 & 2.3 & 5.81 & 1.95 \\
\hline ROA42 & AAT & 4150 & 0.5 & 2.0 & 5.81 & 1.86 \\
\hline ROA43 & $\mathrm{AAT}$ & 3950 & 0.4 & 2.1 & 6.03 & 2.11 \\
\hline ROA46 & AAT & 4050 & 0.5 & 2.2 & 5.83 & 1.91 \\
\hline ROA48 & AAT & 4050 & 0.5 & 2.5 & 5.74 & 1.71 \\
\hline ROA53 & AAT & 3950 & 0.4 & 2.3 & 5.83 & 1.77 \\
\hline ROA58 & AAT & 4200 & 0.6 & 2.2 & 5.77 & 1.76 \\
\hline ROA74 & AAT & 4250 & 0.7 & 2.2 & 5.70 & 1.64 \\
\hline ROA84 & ATT & 3900 & 0.5 & 1.9 & 6.14 & 2.46 \\
\hline ROA94 & AAT & 4200 & 0.7 & 2.3 & 5.72 & 1.67 \\
\hline ROA100 & AAT & 4150 & 0.7 & 2.3 & 6.01 & 2.11 \\
\hline ROA102 & CTIO & 4400 & 1.0 & 3.0 & 5.68 & 1.82 \\
\hline ROA132 & AAT & 3900 & 0.3 & 2.2 & 6.13 & 2.21 \\
\hline ROA144 & AAT & 4200 & 0.8 & 1.8 & 5.84 & 1.94 \\
\hline ROA150 & $\mathrm{AAT}$ & 3950 & 0.6 & 2.2 & 6.25 & 2.41 \\
\hline ROA155 & AAT & 4200 & 0.8 & 2.0 & 5.86 & 1.91 \\
\hline ROA159 & AAT & 4300 & 0.9 & 2.0 & 5.78 & 1.73 \\
\hline ROA161 & AAT & 4250 & 0.8 & 2.1 & 5.83 & 1.91 \\
\hline ROA162 & AAT & 3950 & 0.7 & 2.1 & 6.40 & 2.51 \\
\hline ROA171 & AAT & 4100 & 0.7 & 1.9 & 6.07 & 2.36 \\
\hline ROA179 & AAT & 3850 & 0.5 & 1.5 & 6.40 & 2.51 \\
\hline ROA182 & AAT & 4200 & 0.9 & 2.0 & 6.04 & 2.16 \\
\hline ROA201 & AAT & 3750 & 0.5 & 1.5 & 6.65 & 2.46 \\
\hline ROA209 & CTIO & 4500 & 1.2 & 2.0 & 5.71 & 1.71 \\
\hline ROA213 & CTIO & 4500 & 1.0 & 1.9 & 5.53 & 1.55 \\
\hline ROA219 & CTIO & 3900 & 0.7 & 1.7 & 6.24 & 2.26 \\
\hline ROA231 & AAT & 4000 & 0.7 & 2.2 & 6.40 & 2.41 \\
\hline ROA236 & CTIO & 4200 & 0.7 & 1.7 & 6.05 & 2.16 \\
\hline ROA238 & CTIO & 4550 & 1.2 & 1.7 & 5.70 & 1.83 \\
\hline ROA245 & CTIO & 4300 & 0.7 & 1.8 & 6.08 & 2.14 \\
\hline ROA248 & AAT & 3850 & 0.6 & 1.6 & 6.72 & 2.61 \\
\hline ROA252 & AAT & 4400 & 1.1 & 2.0 & 5.76 & 1.77 \\
\hline ROA253 & CTIO & 4300 & 0.7 & 1.9 & 6.07 & 2.03 \\
\hline ROA279 & AAT & 4350 & 1.1 & 2.0 & 5.81 & 1.81 \\
\hline ROA287 & AAT & 4250 & 1.0 & 1.9 & 6.07 & 2.26 \\
\hline ROA324 & CTIO & 4000 & 0.7 & 1.9 & 6.55 & 2.38 \\
\hline ROA357 & AAT & 4000 & 0.8 & 1.8 & 6.65 & 2.84 \\
\hline ROA371 & AAT & 4000 & 0.9 & 1.6 & 6.71 & 2.81 \\
\hline ROA383 & CTIO & 4400 & 1.0 & 1.8 & 5.87 & 1.99 \\
\hline ROA480 & AAT & 4350 & 1.3 & 1.8 & 6.55 & 2.86 \\
\hline
\end{tabular}

Note.- $\mathrm{A}(\mathrm{X})=\log \epsilon(\mathrm{X})=\log \left(\mathrm{N}_{\mathrm{X}} / \mathrm{N}_{\mathrm{H}}\right)+12$. 


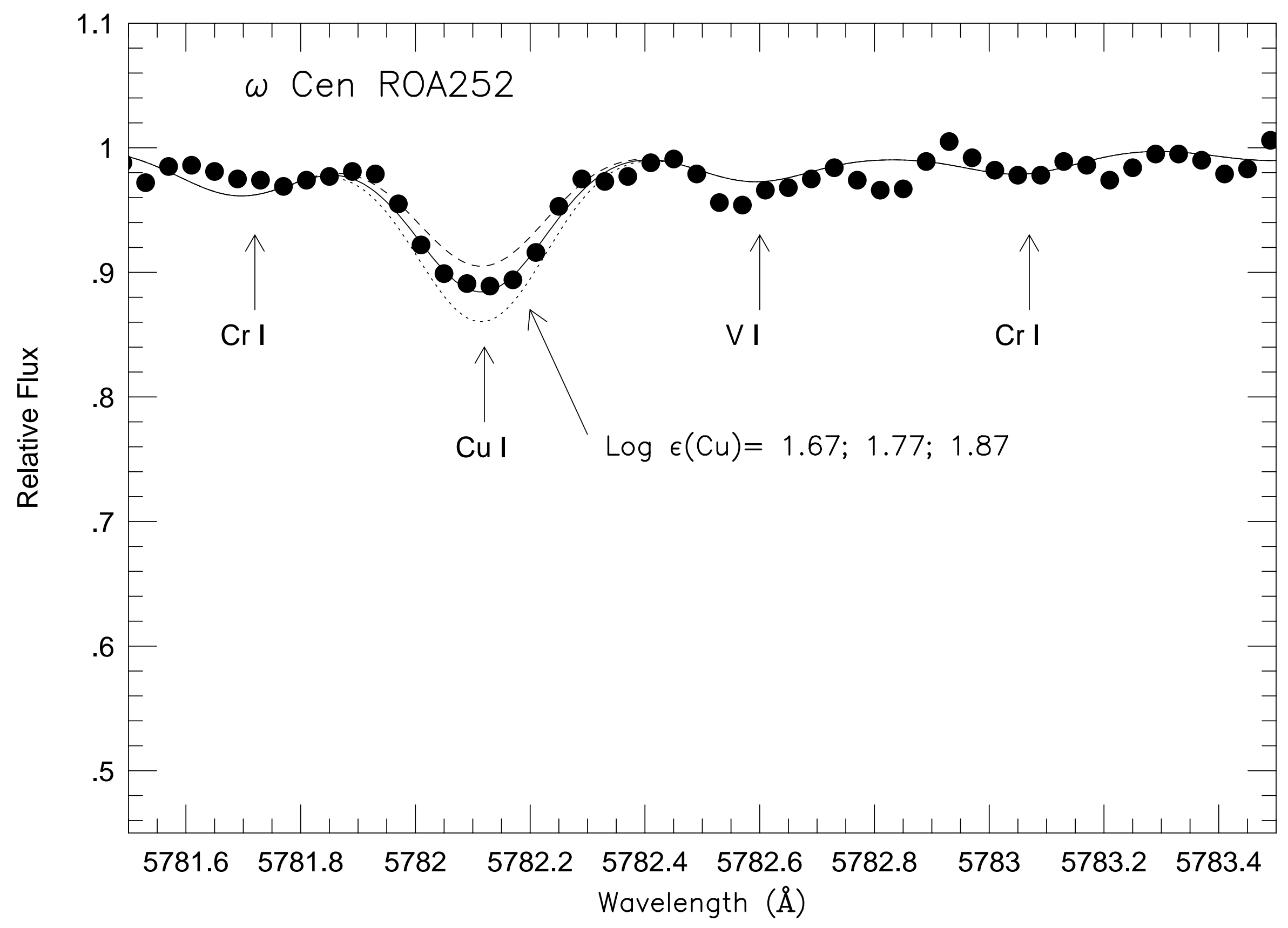


TABLE 4

Iron Equivalent-Width Measurements for NGC288 \& NGC362

\begin{tabular}{|c|c|c|c|c|c|c|c|c|c|c|}
\hline Ior & $\lambda(\AA)$ & $\chi(\mathrm{eV})$ & gf & $288-186$ & 288-199 & $288-231$ & $288-297$ & $362-2213$ & 362 -pe65 & 362 -pe75 \\
\hline \multirow{38}{*}{ Fe I } & 5522.447 & 4.209 & $3.981 \mathrm{e}-02$ & 38 & 36 & 33 & 35 & 44 & 34 & 42 \\
\hline & 5554.895 & 4.548 & $4.169 \mathrm{e}-01$ & - & 74 & 76 & 74 & 86 & 83 & 83 \\
\hline & 5560.212 & 4.434 & $9.120 \mathrm{e}-02$ & 39 & 44 & 33 & 37 & 42 & 48 & 48 \\
\hline & 5569.618 & 3.417 & $3.236 \mathrm{e}-01$ & 137 & 133 & 132 & 138 & 139 & 147 & 137 \\
\hline & 5618.633 & 4.209 & $5.495 \mathrm{e}-02$ & 39 & 38 & 41 & 38 & 48 & 61 & 51 \\
\hline & 5633.947 & 4.991 & $7.586 \mathrm{e}-01$ & 44 & 49 & 53 & 52 & 50 & 63 & 52 \\
\hline & 5635.823 & 4.256 & $1.820 \mathrm{e}-02$ & 24 & 26 & 25 & 25 & 34 & 24 & 24 \\
\hline & 5638.262 & 4.220 & $1.905 \mathrm{e}-01$ & 72 & 72 & 70 & 71 & 75 & 77 & 66 \\
\hline & 5679.023 & 4.651 & $1.698 \mathrm{e}-01$ & 47 & 40 & 38 & 38 & 52 & 55 & 44 \\
\hline & 5691.497 & 4.301 & $4.266 \mathrm{e}-02$ & 34 & 27 & 31 & 35 & 42 & 46 & 46 \\
\hline & 5705.465 & 4.301 & $4.365 \mathrm{e}-02$ & 29 & 28 & 23 & 28 & 32 & 41 & 36 \\
\hline & 5717.833 & 4.284 & $1.047 \mathrm{e}-01$ & 64 & 64 & 66 & 61 & 60 & 76 & 71 \\
\hline & 5806.725 & 4.607 & $1.259 \mathrm{e}-01$ & 28 & 37 & 43 & 34 & 44 & 49 & 40 \\
\hline & 5809.218 & 3.883 & $2.042 \mathrm{e}-02$ & 47 & 51 & 48 & 49 & 57 & 57 & 48 \\
\hline & 5814.808 & 4.283 & $1.514 \mathrm{e}-02$ & 15 & 21 & 27 & 14 & 25 & 26 & 17 \\
\hline & 6024.058 & 4.548 & $8.710 \mathrm{e}-01$ & 91 & 92 & 96 & 94 & 105 & 104 & 80 \\
\hline & 6027.051 & 4.076 & $8.128 \mathrm{e}-02$ & 66 & 65 & 68 & 65 & 72 & 66 & 71 \\
\hline & 6056.005 & 4.733 & $3.981 \mathrm{e}-01$ & 55 & 53 & 53 & 52 & 61 & 63 & 56 \\
\hline & 6079.009 & 4.652 & $1.072 \mathrm{e}-01$ & 22 & 27 & 30 & 28 & 34 & 32 & 34 \\
\hline & 6096.665 & 3.984 & $1.660 \mathrm{e}-02$ & 31 & 30 & 36 & 31 & 35 & 43 & 32 \\
\hline & 6151.618 & 2.176 & $5.129 \mathrm{e}-04$ & 85 & 87 & 89 & 90 & 90 & 105 & 85 \\
\hline & 6157.728 & 4.076 & $7.762 \mathrm{e}-02$ & 67 & 72 & 55 & 70 & 73 & 77 & 70 \\
\hline & 6165.360 & 4.142 & $3.388 \mathrm{e}-02$ & 40 & 39 & 36 & 39 & 45 & 44 & 49 \\
\hline & 6173.336 & 2.223 & $1.318 \mathrm{e}-03$ & 110 & 106 & 100 & 109 & 116 & 109 & 111 \\
\hline & 6187.990 & 3.943 & $2.692 \mathrm{e}-02$ & 41 & 43 & 43 & 42 & 51 & 51 & 47 \\
\hline & 6322.686 & 2.588 & $3.715 \mathrm{e}-03$ & 102 & 100 & 100 & 104 & 110 & 112 & 105 \\
\hline & 6380.743 & 4.186 & $4.786 \mathrm{e}-02$ & 43 & 47 & 50 & 48 & 48 & 47 & 57 \\
\hline & 6393.601 & 2.433 & $3.715 \mathrm{e}-02$ & - & - & 160 & - & 176 & 175 & 158 \\
\hline & 6411.649 & 3.653 & $2.188 \mathrm{e}-01$ & 118 & 116 & 116 & 123 & 128 & 126 & 127 \\
\hline & 6421.351 & 2.279 & $9.772 \mathrm{e}-03$ & 151 & 149 & 155 & 156 & 164 & 162 & 154 \\
\hline & 6430.846 & 2.176 & $9.772 \mathrm{e}-03$ & 158 & 160 & 158 & 162 & 170 & 175 & 160 \\
\hline & 6469.193 & 4.835 & $2.399 \mathrm{e}-01$ & 39 & 44 & 40 & 47 & 50 & 53 & 49 \\
\hline & 6593.871 & 2.437 & $3.802 \mathrm{e}-03$ & 127 & 120 & 124 & 126 & 130 & 127 & 121 \\
\hline & 6597.561 & 4.795 & $1.202 \mathrm{e}-01$ & 28 & 24 & 29 & 20 & 28 & 32 & 28 \\
\hline & 6609.110 & 2.559 & $2.042 \mathrm{e}-03$ & 97 & 98 & 93 & 97 & 110 & 102 & 97 \\
\hline & 6733.151 & 4.637 & $3.715 \mathrm{e}-02$ & 14 & 17 & 11 & 13 & 22 & 22 & 18 \\
\hline & 6820.372 & 4.638 & $6.761 \mathrm{e}-02$ & 24 & 27 & 30 & 33 & 33 & 36 & 32 \\
\hline & 6858.150 & 4.607 & $1.175 \mathrm{e}-01$ & 34 & 34 & 37 & 36 & 40 & 45 & 35 \\
\hline \multirow{5}{*}{ Fe II } & 5991.368 & 3.153 & $2.754 \mathrm{e}-04$ & 30 & 30 & 25 & 29 & 33 & 29 & 36 \\
\hline & 6084.099 & 3.199 & $1.585 \mathrm{e}-04$ & 18 & 17 & 20 & 14 & 21 & 27 & 16 \\
\hline & 6149.246 & 3.889 & $1.905 \mathrm{e}-03$ & 23 & 23 & 19 & 28 & 33 & 32 & 27 \\
\hline & 6416.921 & 3.891 & $2.089 \mathrm{e}-03$ & 25 & 24 & 30 & 26 & 29 & 38 & 32 \\
\hline & 6432.682 & 2.891 & $2.630 \mathrm{e}-04$ & 32 & 32 & 35 & 34 & 38 & 45 & 41 \\
\hline
\end{tabular}

Note.- A '-' indicates an absent or blended line. Equivalent-width units are mÅ. 


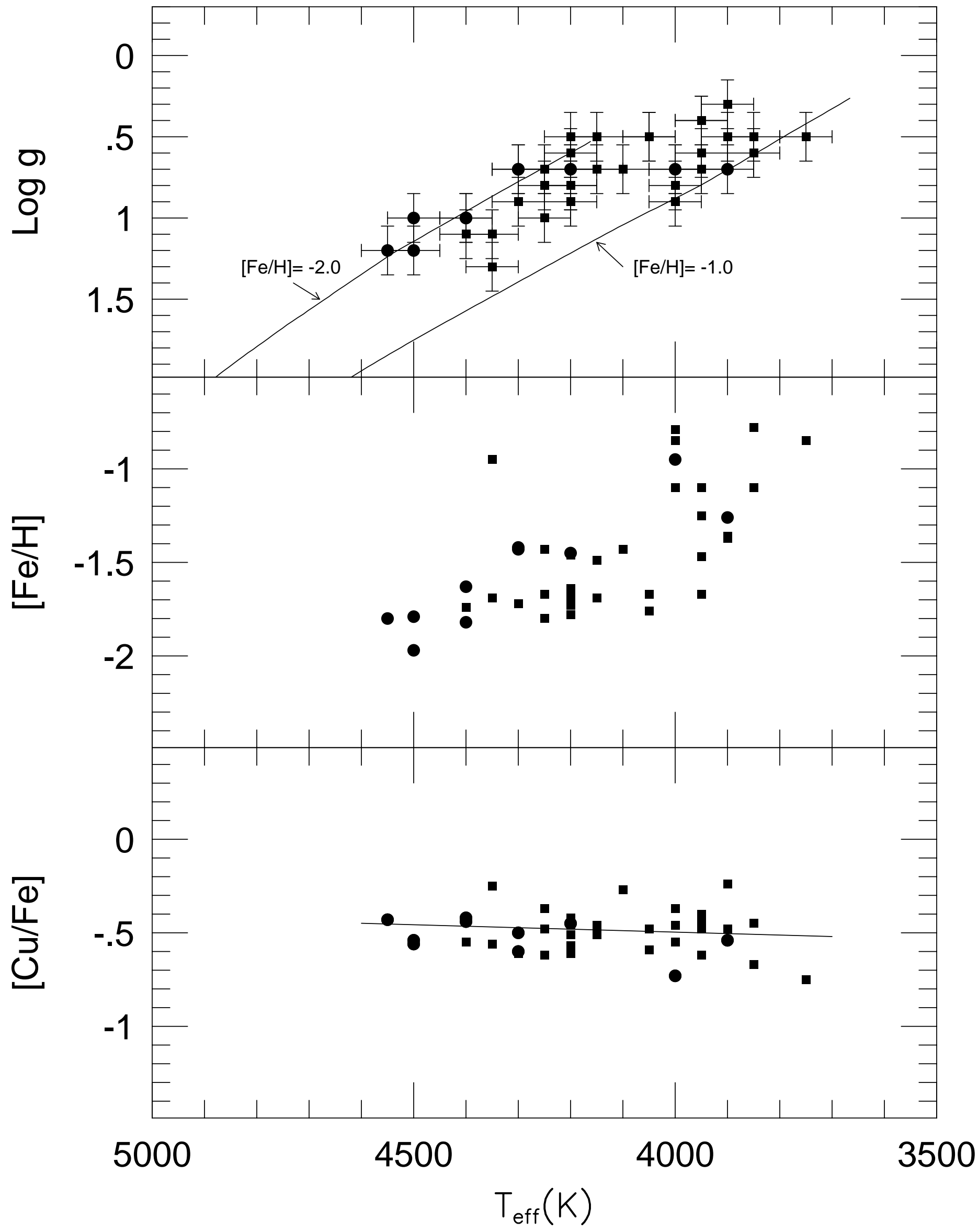


TABLE 5

Iron Equivalent-Width Measurements for M4, NGC3201, \& NGC6752

\begin{tabular}{|c|c|c|c|c|c|c|c|c|c|c|c|}
\hline Io & $\lambda(\AA)$ & $\chi(\mathrm{eV})$ & gf & M4-2519 & M4-2617 & M4-3612 & M4-3624 & $3201-1314$ & $3201-4524$ & 6752-cs3 & $6752-\operatorname{cs} 126$ \\
\hline \multirow[t]{39}{*}{ Fe I } & 5522.447 & 4.209 & $3.981 \mathrm{e}-02$ & 38 & 55 & 46 & 44 & 32 & 22 & 27 & 23 \\
\hline & 5554.895 & 4.548 & $4.169 \mathrm{e}-01$ & 86 & - & 83 & 80 & 75 & 67 & 51 & 58 \\
\hline & 5560.212 & 4.434 & $9.120 \mathrm{e}-02$ & 38 & 52 & 48 & 48 & 31 & 31 & 26 & 31 \\
\hline & 5569.618 & 3.417 & $3.236 \mathrm{e}-01$ & 148 & 145 & 138 & 139 & 133 & 157 & 119 & 142 \\
\hline & 5618.633 & 4.209 & $5.495 \mathrm{e}-02$ & 45 & 55 & 53 & 53 & 40 & 32 & 30 & 33 \\
\hline & 5633.947 & 4.991 & $7.586 \mathrm{e}-01$ & 53 & 57 & 55 & 50 & 44 & 30 & 34 & 31 \\
\hline & 5635.823 & 4.256 & $1.820 \mathrm{e}-02$ & 23 & 31 & 35 & 29 & - & 23 & 16 & 15 \\
\hline & 5638.262 & 4.220 & $1.905 \mathrm{e}-01$ & 72 & 74 & 71 & 77 & 72 & 62 & 55 & 59 \\
\hline & 5679.023 & 4.651 & $1.698 \mathrm{e}-01$ & 48 & 48 & 48 & 44 & 36 & - & 30 & 25 \\
\hline & 5691.497 & 4.301 & $4.266 \mathrm{e}-02$ & 47 & 51 & 41 & 46 & 28 & 37 & 20 & 27 \\
\hline & 5705.465 & 4.301 & $4.365 \mathrm{e}-02$ & 36 & 38 & 34 & 35 & 22 & 23 & 17 & 16 \\
\hline & 5717.833 & 4.284 & $1.047 \mathrm{e}-01$ & 70 & 73 & 78 & 70 & 58 & 51 & 45 & 41 \\
\hline & 5806.725 & 4.607 & $1.259 \mathrm{e}-01$ & 40 & 43 & 44 & 48 & 25 & 32 & 22 & 34 \\
\hline & 5809.218 & 3.883 & $2.042 \mathrm{e}-02$ & 51 & 58 & 55 & 55 & 42 & 41 & 34 & 38 \\
\hline & 5814.808 & 4.283 & $1.514 \mathrm{e}-02$ & 17 & 24 & 26 & 22 & 21 & 16 & 7.5 & 12 \\
\hline & 6016.660 & 3.546 & $2.138 \mathrm{e}-02$ & 86 & - & 96 & 100 & 81 & 61 & 59 & 52 \\
\hline & 6024.058 & 4.548 & $8.710 \mathrm{e}-01$ & 99 & 99 & 97 & 91 & 84 & 99 & 74 & 79 \\
\hline & 6027.051 & 4.076 & $8.128 \mathrm{e}-02$ & 70 & 78 & 68 & 70 & 66 & 57 & 52 & 53 \\
\hline & 6056.005 & 4.733 & $3.981 \mathrm{e}-01$ & 56 & 62 & 60 & 59 & 47 & 52 & 40 & 45 \\
\hline & 6079.009 & 4.652 & $1.072 \mathrm{e}-01$ & - & 41 & 44 & 34 & 22 & 23 & 19 & 21 \\
\hline & 6096.665 & 3.984 & $1.660 \mathrm{e}-02$ & 42 & 38 & 40 & 39 & 32 & 25 & 21 & 25 \\
\hline & 6151.618 & 2.176 & $5.129 \mathrm{e}-04$ & 85 & 94 & 84 & 92 & 92 & 101 & 72 & 82 \\
\hline & 6157.728 & 4.076 & $7.762 \mathrm{e}-02$ & 71 & 82 & 79 & 77 & 71 & 55 & 53 & 53 \\
\hline & 6165.360 & 4.142 & $3.388 \mathrm{e}-02$ & 37 & 47 & 50 & 50 & 36 & 24 & 25 & 26 \\
\hline & 6173.336 & 2.223 & $1.318 \mathrm{e}-03$ & 113 & 113 & 112 & 110 & 120 & 129 & 96 & 114 \\
\hline & 6187.990 & 3.943 & $2.692 \mathrm{e}-02$ & 49 & 54 & 53 & 50 & 42 & 38 & 28 & 33 \\
\hline & 6322.686 & 2.588 & $3.715 \mathrm{e}-03$ & 108 & 112 & 101 & 111 & 111 & 118 & 92 & 111 \\
\hline & 6380.743 & 4.186 & $4.786 \mathrm{e}-02$ & 59 & 62 & 60 & 54 & 41 & 32 & 34 & 33 \\
\hline & 6393.601 & 2.433 & $3.715 \mathrm{e}-02$ & - & - & 160 & 168 & 174 & 205 & 151 & 179 \\
\hline & 6411.649 & 3.653 & $2.188 \mathrm{e}-01$ & 126 & 125 & 120 & 116 & 124 & 133 & 112 & 124 \\
\hline & 6421.351 & 2.279 & $9.772 \mathrm{e}-03$ & 168 & 161 & 156 & 157 & 170 & 193 & 143 & 171 \\
\hline & 6430.846 & 2.176 & $9.772 \mathrm{e}-03$ & 175 & 170 & 171 & 170 & 179 & 200 & 147 & 186 \\
\hline & 6469.193 & 4.835 & $2.399 \mathrm{e}-01$ & 56 & - & 58 & 62 & - & - & 25 & 30 \\
\hline & 6593.871 & 2.437 & $3.802 \mathrm{e}-03$ & 132 & 129 & 125 & 123 & 128 & 142 & 112 & 128 \\
\hline & 6597.561 & 4.795 & $1.202 \mathrm{e}-01$ & 27 & 30 & 31 & 30 & 24 & 14 & 15 & 17 \\
\hline & 6609.110 & 2.559 & $2.042 \mathrm{e}-03$ & 104 & 104 & 104 & 104 & 101 & 106 & 86 & 95 \\
\hline & 6733.151 & 4.637 & $3.715 \mathrm{e}-02$ & 17 & 20 & 22 & 17 & 11 & 14 & 6.5 & 5.0 \\
\hline & 6820.372 & 4.638 & $6.761 \mathrm{e}-02$ & 30 & - & 36 & - & - & - & 16 & 17. \\
\hline & 6858.150 & 4.607 & $1.175 \mathrm{e}-01$ & 45 & 47 & 41 & 42 & - & - & 24 & 24 \\
\hline \multirow[t]{5}{*}{ Fe II } & 5991.368 & 3.153 & $2.754 \mathrm{e}-04$ & 30 & 24 & 26 & 24 & 24 & 33 & 23 & 27 \\
\hline & 6084.099 & 3.199 & $1.585 \mathrm{e}-04$ & 16 & 20 & 17 & 12 & 23 & 26 & 14 & 22 \\
\hline & 6149.246 & 3.889 & $1.905 \mathrm{e}-03$ & 31 & 24 & 22 & 26 & 24 & 35 & 20 & 33 \\
\hline & 6416.921 & 3.891 & $2.089 \mathrm{e}-03$ & 33 & 28 & 29 & 28 & 31 & 30 & 24 & 29 \\
\hline & 6432.682 & 2.891 & $2.630 \mathrm{e}-04$ & 43 & 31 & 29 & 32 & 39 & 46 & 32 & 41 \\
\hline
\end{tabular}

Note.- A '-' indicates an absent or blended line. Equivalent-width units are mÅ. 


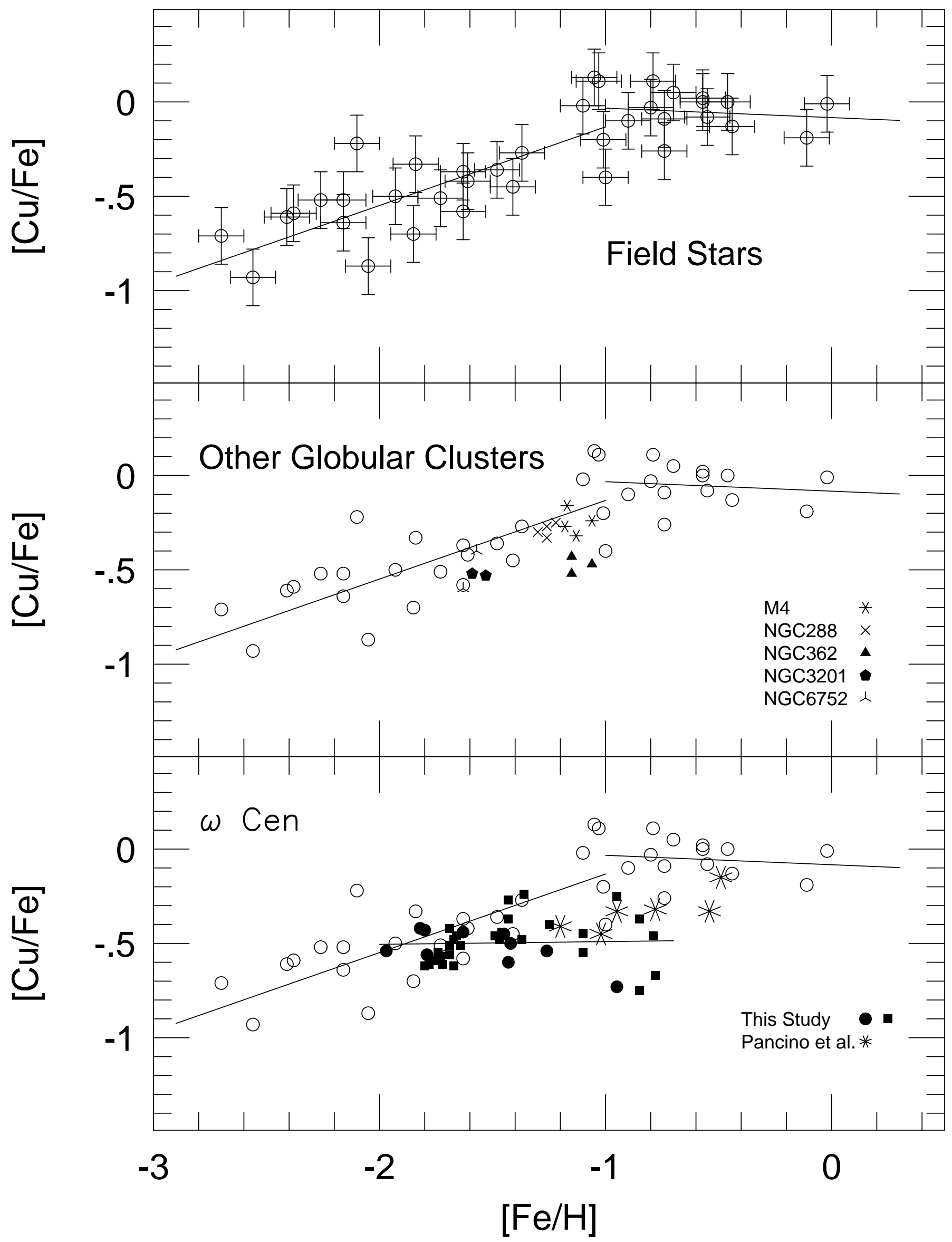


TABLE 6

Globular Cluster Stars: Derived Parameters \& Abundances.

\begin{tabular}{|c|c|c|c|c|c|}
\hline Cluster \& Star & $\mathrm{T}_{\text {eff }}(\mathrm{K})$ & $\log \mathrm{g}\left(\mathrm{cm} \mathrm{s}^{-2}\right)$ & $\xi\left(\mathrm{km} \mathrm{s}^{-1}\right)$ & $\mathrm{A}(\mathrm{Fe})$ & $\mathrm{A}(\mathrm{Cu})$ \\
\hline \multicolumn{6}{|l|}{ M4 } \\
\hline 2519 & 4450 & 1.2 & 1.8 & 6.32 & 2.61 \\
\hline 2617 & 4300 & 1.0 & 1.6 & 6.37 & 2.61 \\
\hline 3612 & 4400 & 1.4 & 1.5 & 6.44 & 2.76 \\
\hline 3624 & 4350 & 1.1 & 1.6 & 6.33 & 2.73 \\
\hline \multicolumn{6}{|l|}{ NGC288 } \\
\hline 186 & 4450 & 1.2 & 1.8 & 6.20 & 2.46 \\
\hline 199 & 4450 & 1.2 & 1.7 & 6.24 & 2.47 \\
\hline 231 & 4550 & 1.4 & 1.8 & 6.28 & 2.59 \\
\hline 297 & 4500 & 1.4 & 1.9 & 6.24 & 2.53 \\
\hline \multicolumn{6}{|l|}{ NGC362 } \\
\hline 2213 & 4450 & 1.2 & 1.7 & 6.35 & 2.48 \\
\hline pe65 & 4550 & 1.4 & 1.8 & 6.44 & 2.53 \\
\hline pe75 & 4500 & 1.4 & 1.7 & 6.35 & 2.39 \\
\hline \multicolumn{6}{|l|}{ NGC3201 } \\
\hline 1314 & 4250 & 0.5 & 2.1 & 5.97 & 2.01 \\
\hline 4524 & 4300 & 0.3 & 2.6 & 5.91 & 1.94 \\
\hline \multicolumn{6}{|l|}{ NGC6752 } \\
\hline $\operatorname{cs} 3$ & 4450 & 1.2 & 2.0 & 5.93 & 2.09 \\
\hline cs126 & 4200 & 0.2 & 2.0 & 5.87 & 1.83 \\
\hline
\end{tabular}

Note.- Solar abundances taken as $\mathrm{A}(\mathrm{Fe})=7.50$ and $\mathrm{A}(\mathrm{Cu})=4.06 . \quad \mathrm{A}(\mathrm{X})=\log \epsilon(\mathrm{X})=$ $\log \left(\mathrm{N}_{\mathrm{X}} / \mathrm{N}_{\mathrm{H}}\right)+12$. 